\title{
fMRl-compatible registration of jaw movements using a fiber-optic bend sensor
}

\author{
Peter Sörös ${ }^{1}{ }^{*}$, Bradley J. MacIntosh ${ }^{1,2}$, Fred Tam ${ }^{1,3}$ and Simon J. Graham ${ }^{1,3,4,5}$ \\ 1 Department of Imaging Research, Sunnybrook Health Sciences Centre, Toronto, Ontario, Canada \\ 2 Functional Magnetic Resonance Imaging of the Brain Centre, University of Oxford, Oxford, UK \\ 3 Rotman Research Institute, Baycrest, Toronto, ON, Canada \\ ${ }^{4}$ Department of Medical Biophysics, University of Toronto, Toronto, ON, Canada \\ ${ }^{5}$ Heart and Stroke Foundation of Ontario Centre for Stroke Recovery, Ottawa, ON, Canada
}

\section{Edited by:}

Srikantan S. Nagarajan,

University of California, San Francisco, USA

\section{Reviewed by:}

Lee M. Miller,

University of California, Davis, USA

John F. Houde,

University of California, San Francisco,

USA

${ }^{*}$ Correspondence:

Peter Sörös,

Department of Communication

Sciences and Disorders,

University of South Carolina, 915

Greene Street, Columbia, SC 29208

USA.

e-mail:peter.soros@gmail.com
A functional magnetic resonance imaging (fMRI)-compatible fiber-optic bend sensor was investigated to assess whether the device could be used effectively to monitor opening and closing of the jaw during an $\mathrm{fMRl}$ experiment at $3 \mathrm{~T}$. In contrast to surface electromyography, a bend sensor fixed to the chin of the participant is fast and easy to use and is not affected by strong electromagnetic fields. Bend sensor recordings are characterized by high validity (compared with concurrent video recordings of mouth opening) and high reliability (comparing two independent measurements). The results of this study indicate that a bend sensor is able to record the opening and closing of the jaw associated with different overt speech conditions (producing the utterances /a/, /pa/, /pataka// and the opening of the mouth without speech production. Data post-processing such as filtering was not necessary. There are several potential applications for bend sensor recordings of speech-related jaw movements. First, bend sensor recordings are a valuable tool to assess behavioral performance, such as response latencies, accuracies, and completion times, which is particularly important in children, seniors, or patients with various neurological or psychiatric conditions. Second, the timing information provided by bend sensor data may improve the predicted hemodynamic response that is used for $\mathrm{fMRI}$ analysis based on the general linear model (GLM). Third, bend sensor recordings may be included in GLM analyses not for statistical contrast purposes, but as a covariate of no interest, accounting for part of the data variance to model fMRI artifacts due to motion outside the field of view.

Keywords: bend sensor, functional magnetic resonance imaging, speech production, articulation, oro-facial movement

\section{INTRODUCTION}

In recent years, a growing number of functional magnetic resonance imaging (fMRI) studies on the neural correlates of overt speaking (Abrahams et al., 2003; Gracco et al., 2005; Bohland and Guenther, 2006; Sörös et al., 2006a; Christoffels et al., 2007; Riecker et al., 2008) and singing (Riecker et al., 2000; Kleber et al., 2007) have been published. A major advantage of investigating overt speech in contrast to silent (or covert) speech is the possibility to monitor the behavioral performance during the experiment (Abrahams et al., 2003). Assessing task performance such as response latency or movement amplitude is especially important in studies involving children, seniors, or patients with various neurological or psychiatric conditions who might give delayed responses or even omit responses.

The objective of the present study was to demonstrate the use of a fiber-optic bend sensor, fixed to the chin, to record speech-related jaw movements during fMRI. Fiber-optic based bend sensors have been used in various fields of biomedical research, including the monitoring of finger (Ku et al., 2003; Gorbet et al., 2004; Jindrich et al., 2004; Gorbet and Sergio, 2007) and ankle movements (Seto, 2000; Seto et al., 2001; MacIntosh et al., 2004).

\section{LIMITATIONS OF CURRENT TECHNIQUES}

To study speech-related movements outside MRI systems, surface electromyography (EMG) is widely used (Smith, 1992). In positron emission tomography (Murphy et al., 1997) and magnetoencephalography (Sörös et al., 2003), EMG has also been used frequently and successfully to monitor muscle function associated with overt speech production. The use of EMG in fMRI studies, however, is challenging (for a recent review on the recording of electrophysiological data during fMRI, see Laufs et al., 2008). Time-varying radiofrequency pulses and magnetic field gradients may induce artifacts in EMG recordings and, conversely, movements of EMG electrodes and leads inside the magnetic field may cause artifacts in fMRI data (Ganesh et al., 2007; MacIntosh et al., 2007). In addition, the use of EMG in an fMRI study is limited by the need of an fMRI-compatible EMG system (e.g., BrainAmp, Brain Products, Gilching, Germany ${ }^{1}$ ), specialized EMG data postprocessing (van Duinen et al., 2005; MacIntosh et al., 2007), and by time constraints. Setting up a combined fMRI-EMG experiment requires extra time for the preparation of the skin, the mounting of the electrodes and the checking of the electrode impedance.

'http://www.brainproducts.com 
Increasing the length of an fMRI experiment is especially problematic in studies involving participants for whom the monitoring of behavioral performance is most important, such as the populations named above.

To record high-quality acoustic signals associated with overt speech production during an fMRI experiment, noise cancelling optical microphones have been utilized (e.g., FOMRI II microphone, Optoacoustics Ltd., Or-Yehuda, Israel ${ }^{2}$ ). These microphones reduce the intense acoustic noise, often exceeding $100 \mathrm{~dB}$ SPL, during fMRI experiments by real-time adaptive noise cancelling (Chambers et al., 2007) and can be used even during continuous scanning. Acoustic recordings of overt responses are essential for the assessment of response accuracy, but do not represent oro-facial movements.

To record oro-facial movements associated with speech production, MRI-compatible cameras have been used successfully (e.g., MRC Systems, Heidelberg, Germany ${ }^{3}$ ) (Graham et al., 2009). Such cameras, which must be integrated with a dedicated video capture system, can be difficult to mount in the magnet bore without obstructing the patient and head coil as they move to and from isocenter, and require appropriate line-of-sight from the camera to the mouth through apertures in the head coil. To determine the onset and the amplitude of movements based on video recordings, extensive image processing is also required.

\section{MATERIALS AND METHODS BEND SENSOR}

Bend sensor recordings were performed using a flexible, fMRIcompatible fiber-optic bend sensor (S700 ShapeSensor, Measurand Inc., Fredericton, NB, Canada ${ }^{4}$ ) that allows one degree of freedom measurements (Figure 1) (Seto et al., 2001).

${ }^{2} \mathrm{http}: / /$ www.optoacoustics.com

${ }^{3}$ http://www.mrc-systems.de

${ }^{4} \mathrm{http}: / /$ www.measurand.com/products/ShapeSensor.html

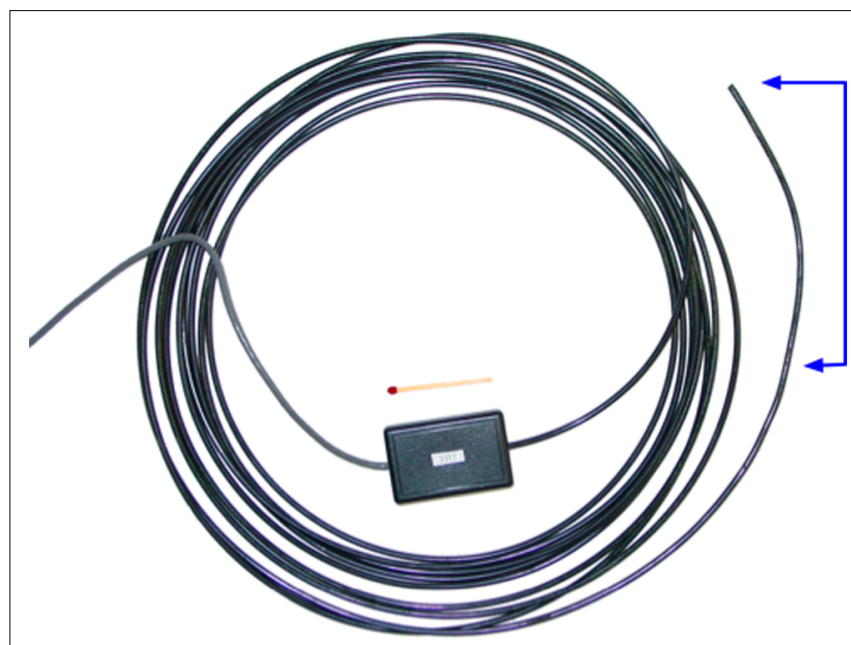

FIGURE 1 |The fiber-optic bend sensor to monitor jaw movements. The active zone of the sensor (right, indicated in blue) is attached to the participant's chin. The electronics (middle) are located outside the magnet room and consist of an LED and two photodiodes for transmission and detection of the light, respectively. A matchstick is shown for scale.
This sensor contains a single strand plastic optical fiber (transmit and return paths, diameter $=0.25 \mathrm{~mm}$ ) encased in a plastic cylindrical sheath of sufficient length (i.e., $6 \mathrm{~m}$ ) to route from the operator console to the center of the magnet bore through an appropriately located wave guide in the radiofrequency shield enclosing the magnet room. The reflectiveness of one side of the optical fiber cladding is purposely degraded in the "U-shaped" active zone within the cylindrical sheath, such that attenuation of light in the fiber is proportional to how much the fiber is bent. Bend sensor signals were recorded with an associated electronics box, consisting primarily of one light emitting diode (LED) for transmission and two photodiodes for signal reception, which was located near the operator console outside the magnet room.

\section{CHARACTERISTICS OF THE BEND SENSOR SIGNAL}

To determine the warm-up characteristics of the bend sensor, the sensor was fixed in position outside the magnet. Measurements of the sensor signal were acquired (USB-6008, National Instruments, Austin, TX, USA) at $400 \mathrm{~Hz}$ for $20 \mathrm{~min}$.

To assess the stability of bend sensor measurements after the warmup phase, the bend sensor was fixed in place and allowed to warm up for over $30 \mathrm{~min}$. The sensor signal was then sampled at $400 \mathrm{~Hz}$ for $5 \mathrm{~min}$ to assess stability. Measurements were conducted inside the magnet during a routine $\mathrm{fMRI}$ scan (EPI, TR/TE/FA $=2000 \mathrm{~ms} /$ $30 \mathrm{~ms} / 70^{\circ}, 64 \times 64$ matrix, $200 \mathrm{~mm}$ FoV, 28 axial slices, $5 \mathrm{~mm}$ thick), with a phantom, in a 3-T MR imager (Magnetom Tim Trio, Siemens, Erlangen, Germany). The tip of the sensor was about $35 \mathrm{~cm}$ from isocenter such that it was possible to position and manipulate the sensor tip appropriately by reaching into the magnet bore and without moving the patient table, which would disrupt scanning.

To assess the dynamic performance and MRI-compatibility of the bend sensor, the sensor tip was manually slid between two points in space that were $2 \mathrm{~cm}$ apart on a ruler to simulate a moderate mouth movement, using music for temporal reference. Movements were repeated at two speeds (approximately 0.25 and $0.5 \mathrm{~Hz}$ ), both inside the magnet and outside the MRI room. Inside-magnet measurements were conducted during a routine fMRI scan (see above).

To assess the reliability of the bend sensor signal, a Pearson correlation coefficient was computed between these two independent bend sensor recordings (inside the magnet and outside the MRI room).

To study the validity of bend sensor recordings, the relationship between the bend sensor signal and mouth movements was assessed. The bend sensor was taped to the chin of a volunteer (Figure 2) and allowed to warm up for several minutes. The signal

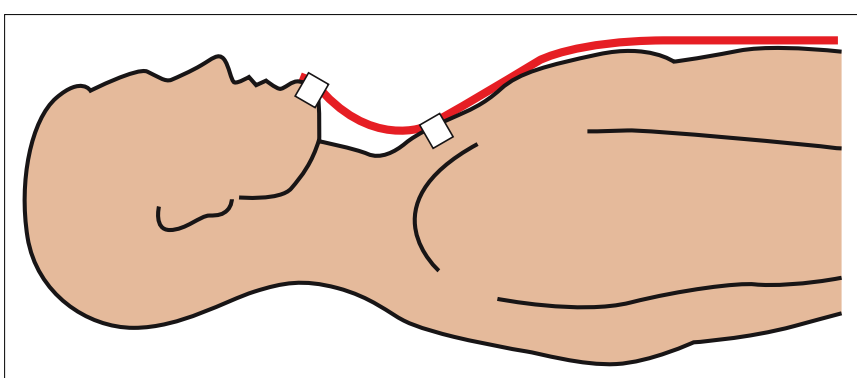

FIGURE 2 | Mounting of the bend sensor. The sensor (red) is fixed to the chin and chest of the participant with adhesive tape (3M Durapore, white). 
was measured at $400 \mathrm{~Hz}$, and an MRI-safe video camera (12M, MRC Systems GmbH, Heidelberg, Germany) in conjunction with a specially developed video capture system (Graham et al., 2009) was used to record video at $30 \mathrm{~Hz}$ within the magnet bore. An electrical trigger was used to synchronize the sensor and video recordings before the volunteer performed several mouth movements. The video analysis consisted of manually selecting the center of a tape mark on the chin in each video frame and noting the pixel location.

\section{BEND SENSOR RECORDINGS DURING OVERT SPEECH PRODUCTION Participants}

To study jaw movements in an actual fMRI experiment involving overt speech production, bend sensor signals were recorded in seven healthy younger volunteers (three women, four men; mean age $=25$ years) and in eight healthy older adults (four women, four men; mean age $=70$ years). All participants were right-handed and fluent speakers of English. The study was approved by the Research Ethics Board at Sunnybrook Health Sciences Centre. Informed consent for participation in the project was obtained from all subjects according to the Declaration of Helsinki. The present study is part of a broader project investigating the neural correlates of overt speech production (Sörös et al., 2006a,b, 2009).

\section{Tasks and fMRI data acquisition}

Subjects were asked to repeat acoustically presented sub-lexical speech sounds of different complexity and to perform oral movements without articulation. The required responses were the long vowel /a/, a consonant-vowel syllable (/pa/), a three-syllabic utterance (/pataka/), and oral movements (opening the mouth or protruding the lips). Instructions were delivered through the audio Silent Scan Audio System at a constant onset-to-onset interstimulus interval of $10 \mathrm{~s}$ using padded headphones with a noise reduction rating of $30 \mathrm{~dB}$ to reduce acoustic fMRI noise. Subjects were asked to perform the given task or articulate the required response immediately after the end of the verbal instruction. Six experimental sessions were performed. Each session comprised six separate blocks of speech (five events per block, $50 \mathrm{~s}$ duration each), two blocks of oral movement (five events, 50 s duration each) and three blocks of baseline (30 s, no verbal cues). Blocks were grouped in a pseudo-randomized order with sessions 1, 3, 5 and 2, 4, 6 having the same order. For fMRI, clustered image acquisition (sparse temporal sampling) was performed (Edmister et al., 1999). All instructions were delivered and all responses were made within the silent interval between the acquisition of the fMR images thus separating the hemodynamic brain activation signals associated with acoustic fMRI noise from the speech-related behaviors of interest. The experiment is illustrated in Figure 3. For a discussion of the details

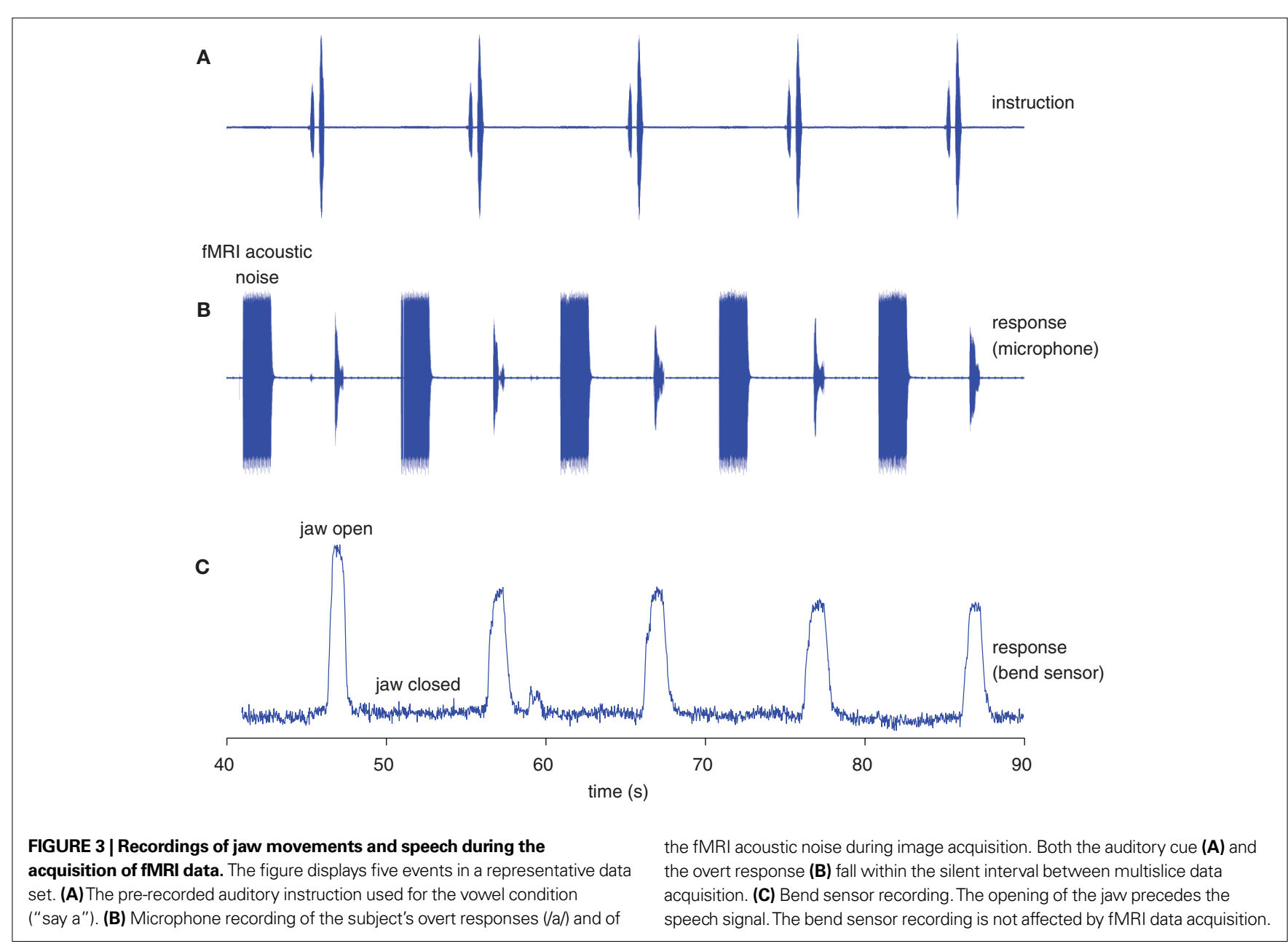


of the experimental setup, imaging parameters, and the subsequent functional brain maps the reader is referred to previous papers of our group (Sörös et al., 2006a, 2009).

\section{Bend sensor recordings}

The sensor was attached to the chin and the chest of each participant immediately before the fMRI experiment using adhesive tape (Figure 2). Paper tape (3M, St. Paul, MN, USA) was used in the initial experiments, which was not adhesive enough and led to a dislocation of the sensor tip at the chin. Medical silk tape (3M Durapore, St. Paul, MN, USA), in contrast, was used in all following measurements and provided sufficient adhesion.

Bend sensor signals were digitized at $40 \mathrm{~Hz}$ and recorded using a custom-written Labview program (National Instruments, Austin, TX, USA ${ }^{5}$. Collection of bend sensor and fMRI data were synchronized by the stimulation software E-Prime (Psychology Software Tools, Pittsburgh, PA, $\mathrm{USA}^{6}$ ). The onset latency and the amplitude of jaw opening were calculated using a custom-written program for the statistical package $\mathrm{R}^{7}$. Bend sensor recordings were baseline corrected and normalized to the largest amplitude in the entire session. The relative peak amplitude and the onset of jaw opening were determined for each epoch separately. To assess the onset of jaw opening, the mean and standard deviation (SD) of the data points prior to the first instruction in each experimental run were calculated to quantify the bend sensor signal baseline. The length of the baseline was 1600 points (40 s). Task timing details are given below. Movement onset was defined as the time point at which the signal exceeded the mean value of the baseline +5 SD. For comparison between jaw movements and the acoustic speech signal, the participants' overt responses were recorded via the microphone channel of the fMRI-compatible Silent Scan Audio System (Avotec, Stuart, FL, USA ${ }^{8}$ ), digitized at $44.1 \mathrm{kHz}$, and stored as an audio file on a PC.

\section{RESULTS}

\section{CHARACTERISTICS OF THE BEND SENSOR SIGNAL}

During the warm-up phase with the sensor fixed, the signal drifts slowly by about $4 \%$ over approximately $15 \mathrm{~min}$ (Figure 4). After the warm-up phase, the SD of the signal is $1.56 \%$ (inside the magnet) and $1.86 \%$ (outside the magnet room) during a 5-min recording (sampling rate $400 \mathrm{~Hz}, 120,000$ data points; data not shown).

A comparison of bend sensor recordings inside the magnet and outside the magnet room with different frequencies $(0.25$ and $0.5 \mathrm{~Hz}$ ) revealed no artefacts or distortions of the sensor signal due to fMRI scanning (Figure 5).

A Pearson correlation was performed between these bend sensor recordings (Figure 5). For an alpha level of 0.05, the correlation between the two recordings at a frequency of $0.25 \mathrm{~Hz}$ was found to be statistically significant $[r(7998)=0.98, p<0.0001$, $\left.R^{2}=0.96\right]$. A statistically significant correlation was also found between two recordings at a higher frequency of approximately $0.5 \mathrm{~Hz}\left[r(7998)=0.92, p<0.0001, R^{2}=0.84\right]$.

${ }^{5}$ http://www.ni.com/labview/

${ }^{6}$ http://www.pstnet.com/products/e-prime/

${ }^{7} \mathrm{http}: / /$ www.r-project.org

${ }^{8} \mathrm{http}: / /$ www.avotec.org/silentscan.htm

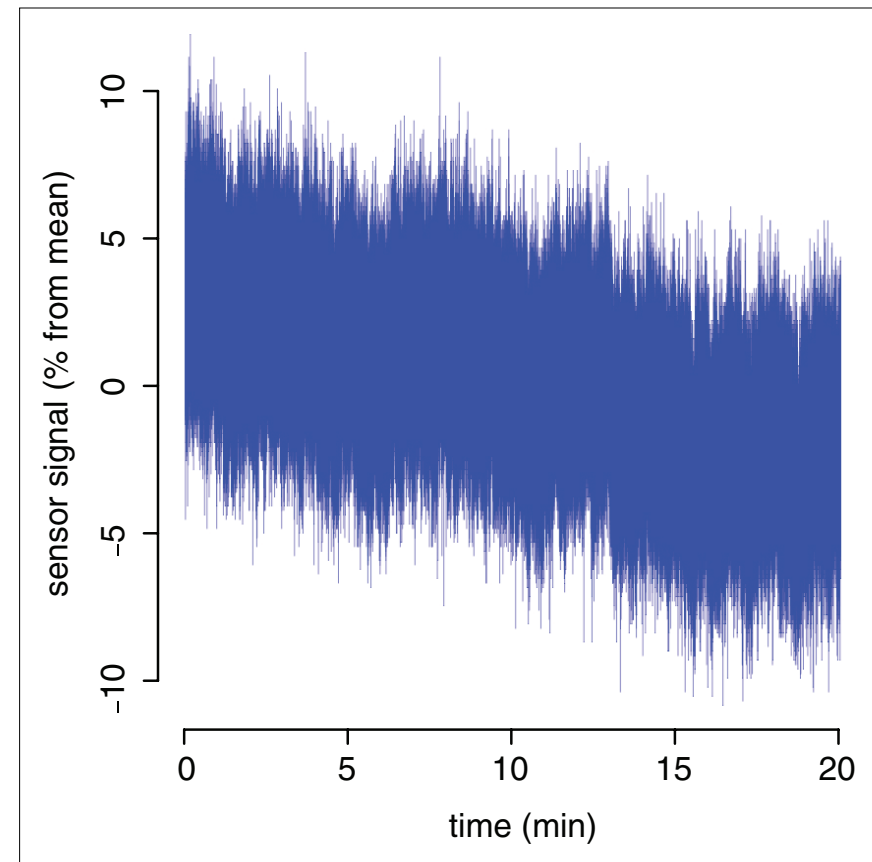

FIGURE 4 |Warm-up characteristics of the bend sensor signal.

A Pearson correlation addressed the relationship between mouth opening as determined by the analysis of a video recording of chin movement and the concurrent bend sensor signal (Figure 6). For an alpha level of 0.05 , the correlation between mouth opening and sensor signal was found to be statistically significant $\left[r(164)=0.94, p<0.0001, R^{2}=0.89\right]$. Figure 6 also shows that the onset and offset of mouth movement are measured very similarly by both recordings.

\section{BEND SENSOR RECORDINGS DURING OVERT SPEECH PRODUCTION}

The MRI-compatible bend sensor was able to record the opening and closing of the jaw for all overt speech conditions (/a/,/pa/,/pataka/) used in this experiment. Bend sensor recordings from 6 individual participants are shown in Figure 7. In two initial measurements, however, the sensor was dislocated during the experiment, probably due to perspiration and an insufficiently adhesive tape. In the movement condition, two different oral movements were required, opening the mouth and protruding the lips without mouth opening. Mouth opening without speech production was also associated with a reliable signal in all participants, often with the highest amplitude across all conditions. As expected, protruding the lips did not reveal consistent signals (Figure 7). Figure 8 illustrates the consistency of bend sensor recordings between blocks of responses for one condition (here: /a/ condition, data of one participant). Inter-individual variability of the onset of the bend sensor signal during opening of the jaw is shown in Figure 9. Signal onset times were not significantly different between younger and older participants.

\section{DISCUSSION}

In many speech production experiments, monitoring the behavioral performance of the participants is desired. Outside the scanner, recording of speech-related muscle activity and of overt speech 

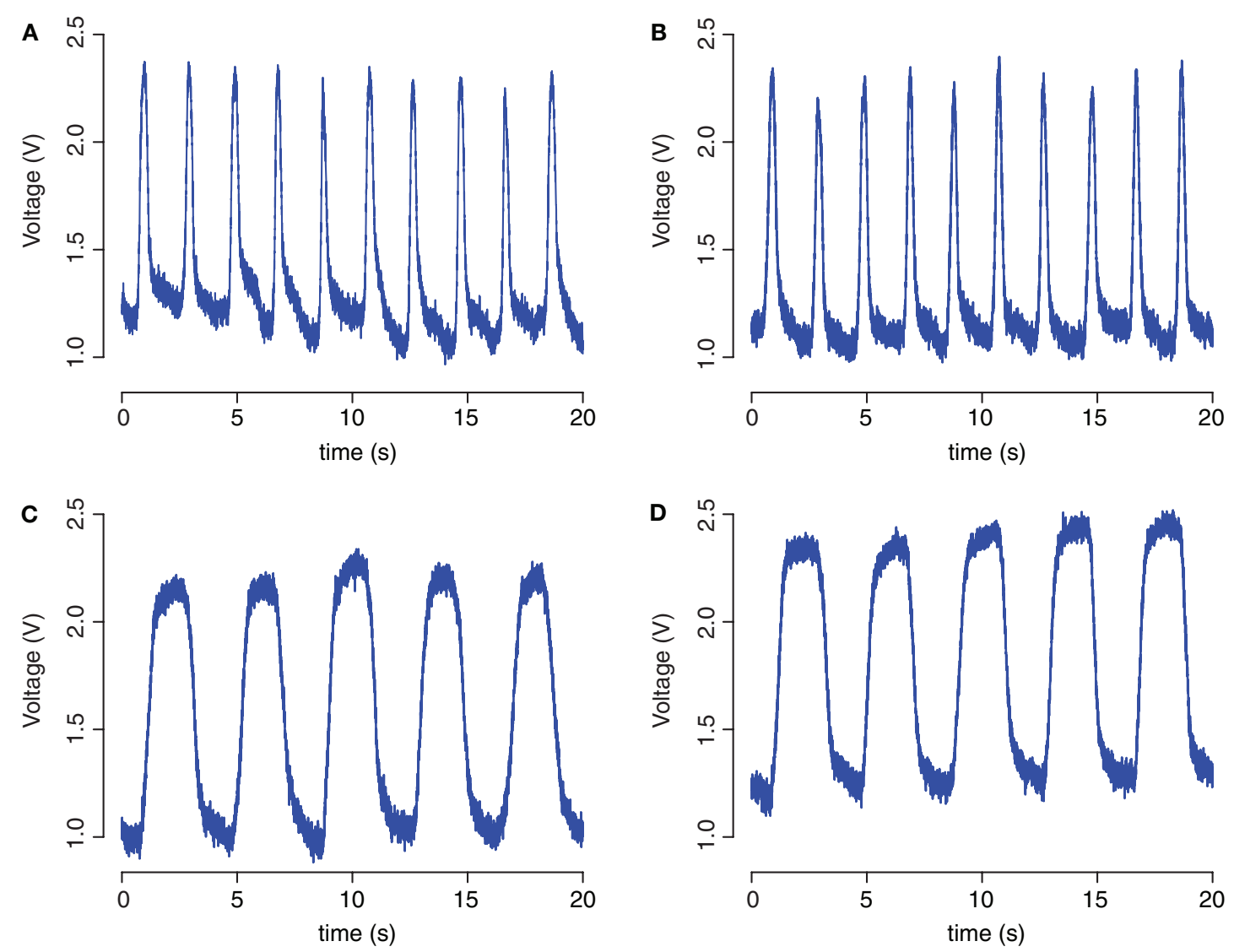

FIGURE $\mathbf{5}$ | Bend sensor recordings during manual movements. Recordings were performed outside the magnet room $(\mathbf{A}, \mathbf{C})$ and inside the magnet $(\mathbf{B}, \mathbf{D})$ with a frequency of approximately $0.5 \mathrm{~Hz}(\mathbf{A}, \mathbf{B})$ and $0.25 \mathrm{~Hz}(\mathbf{C}, \mathbf{D})$.

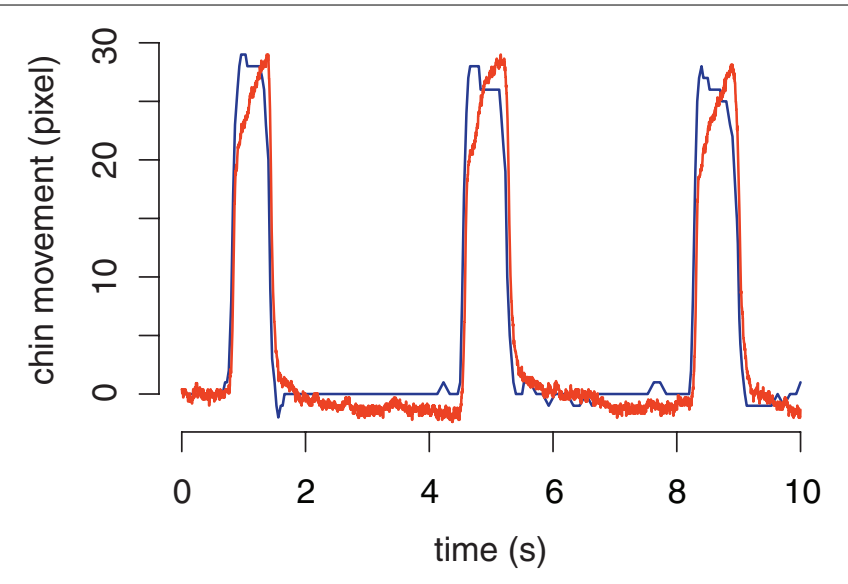

FIGURE 6 | Comparison of a video recording and a concurrent bend sensor recording of mouth opening. The blue curve represents the opening and closing of the mouth, determined with an fMRI-safe video camera. The red curve represents the bend sensor signal in arbitrary units.

can be easily performed using surface EMG and a microphone connected to a computer. Inside the scanner, the use of these techniques is limited by the acoustic noise and the strong timevarying electromagnetic fields during fMRI (Ganesh et al., 2007). In this study, recording the opening and closing of the jaw using a fiber-optic, fMRI-compatible bend sensor proved to be a valuable alternative to the established methods, demonstrating good reliability and validity (Figures 5 and 6). Bend sensor recordings were found to be valid as assessed by a comparison between video recordings and concurrent bend sensor recordings of mouth opening (Figure 6). There was a close agreement between the onset of mouth opening in both methods, emphasizing the usefulness of the bend sensor to monitor response latencies. The shape of the bend sensor signal curve, however, showed some slight differences compared to the analysis of the video recording, particularly a more rounded time course during the period when the mouth was most open. These differences could be due to several effects. First, the sensor signal is sensitive to bend angle and does not record linear displacements. Consequently, a deviation would be expected between bend sensor signals and displacement data, when displacements become large. However, in Figure 6 the bend sensor signals continue to increase toward a signal plateau during periods when the video data record a quite consistent displacement indicative of holding the mouth open in a fixed position. This is more likely explained by the fact that the bend angle and thus the sensor signal is influenced not only by the actual mouth movement but also by the fixation of the sensor and its potential movement when being bent. Parameters that influence the fixation of the sensor include the adhesiveness of 

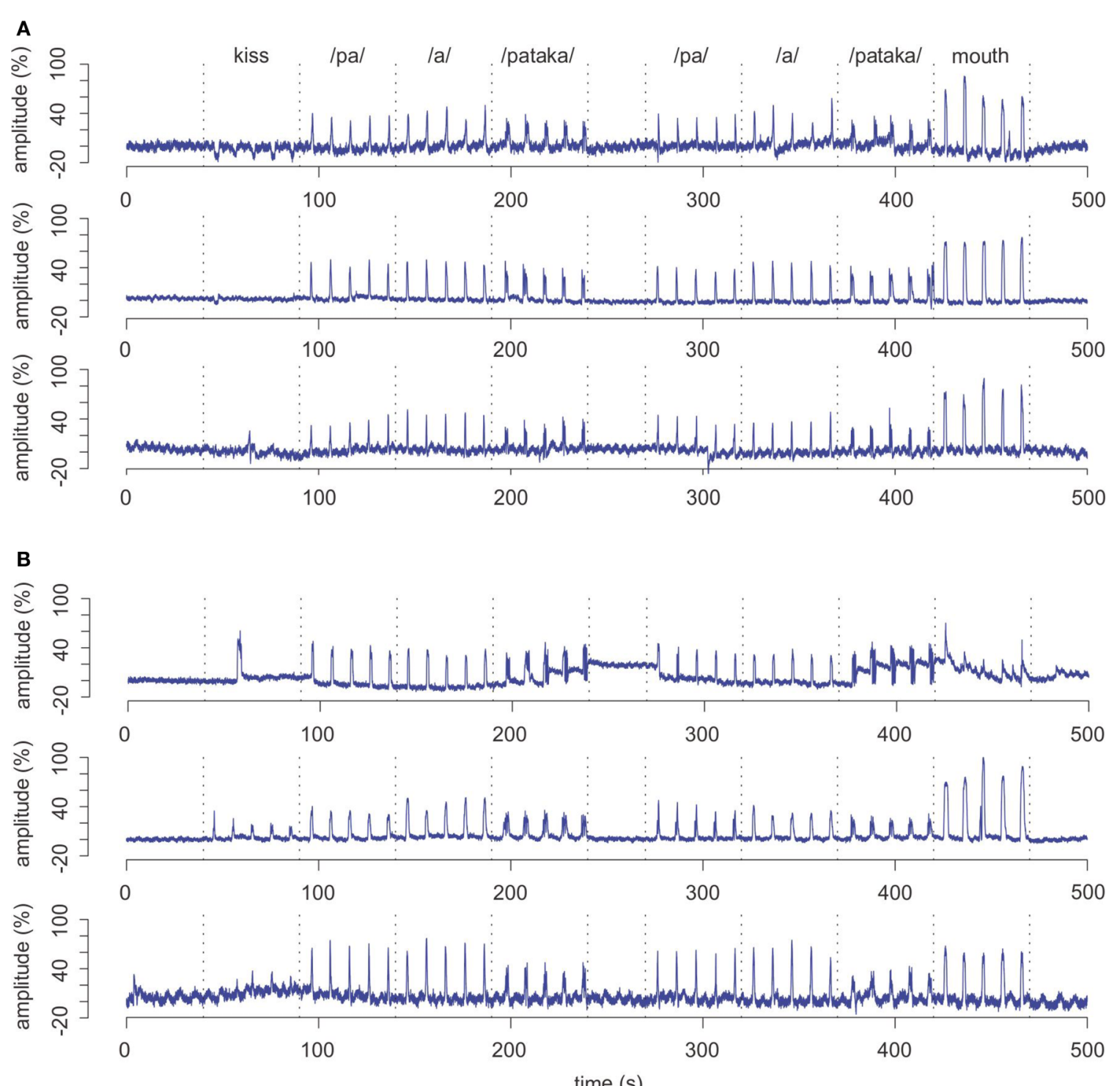

FIGURE 7 | Bend sensor recording of 6 individual participants. Data from one run of three younger $(\mathbf{A})$ and three older individuals $(\mathbf{B})$ are shown in blue. In the runs shown here, the participants performed all verbal tasks correctly as verified by the concurrent audio recording. All bend sensor recordings are baseline-corrected $(0-40$ s prior to the beginning of the first block) and normalized to the largest deflection in the entire data set which was set to a relative amplitude of $100 \%$. The dotted lines mark the beginning and end of blocks of five responses. Kiss denotes protruding the lips. Mouth denotes opening the mouth without producing an utterance. the tape used, the angle of the sensor tip relative to the chin, the participant's skin surface environment and the condition of the participant's clothing on which the sensor is fixed.

The bend sensor assesses the opening and closing of the mouth, which appears to be more closely related to the activation of cortical and subcortical cognitive and motor areas than voice onset. In a variety of sounds, mouth movements can start substantially earlier than sound production, e.g., in the stop consonant $/ \mathrm{p} /$. Thus, the bend sensor signal is useful to determine the response latency for mouth movement, but, in general, cannot be used to differentiate between the mouth movements associated with different sounds produced during an experiment. It has to be noted that the onset of the bend sensor signal showed considerable inter-individual variability (Figure 9), which should be minimized to improve comparisons across conditions or subject groups. Subject variability could be reduced by giving an explicit instruction ("respond as rapidly as possible with equal amplitude") and adding a short training session before starting with the actual experiment.

The preparation of the volunteer, in particular fixing the sensor with adhesive tape and testing the signal, was considerably faster than the preparation usually needed for EMG studies. The bend sensor, however, has to be carefully fixed at the chin. Probably due to perspiration and insufficiently adhesive paper tape, the sensor was dislocated during two of the initial measurements. In the following experiments, the sensor was fixed with silk tape without dislocation 

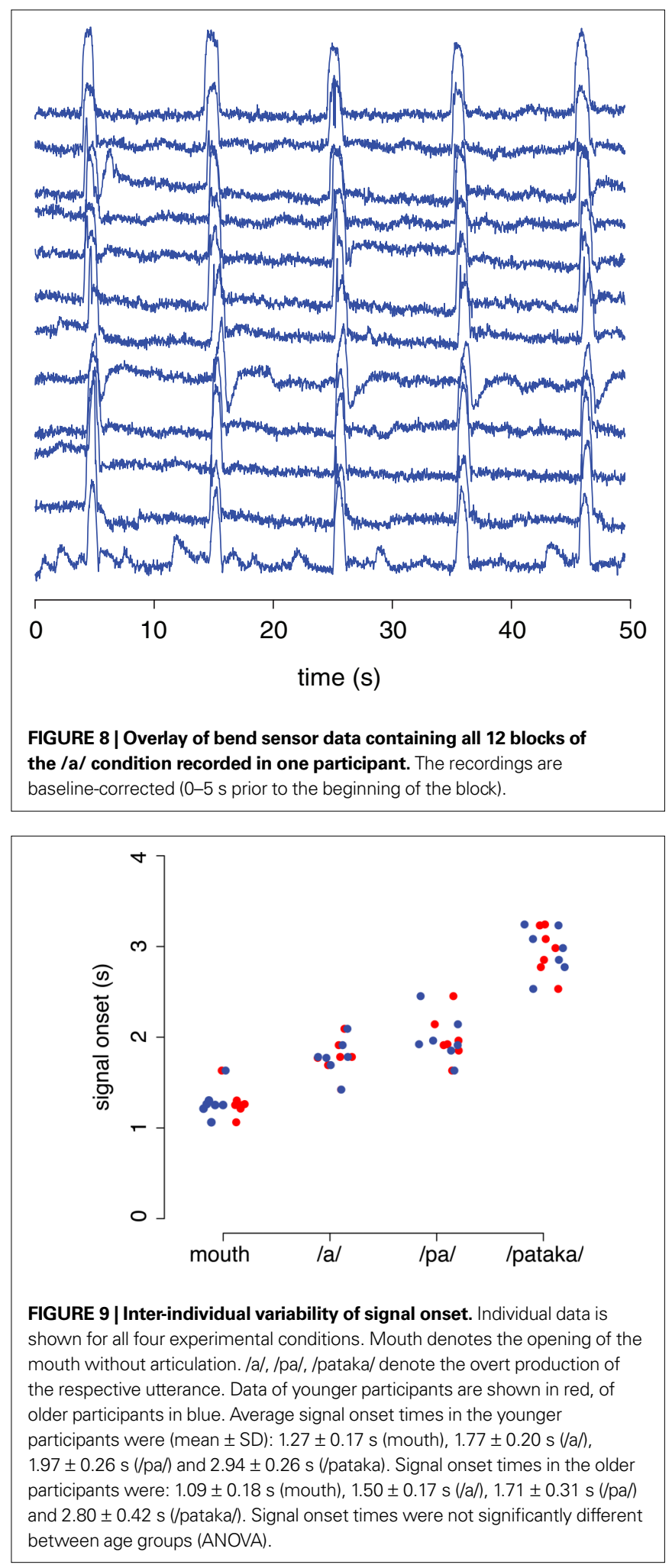

during the entire measurement. To ensure a tight contact between the adhesive tape and the skin, the participants should be asked to wash or shave their chin before the experiment. In addition, participants should wear light clothing to avoid excessive perspiration. In contrast to EMG (Ganesh et al., 2007), Figure 5 indicates that
fMRI data acquisition has no impact on the quality of bend sensor recordings. Previous work has shown that use of fiber-optic bend sensing technology has no statistically significant impact on fMRI data quality (Di Diodato et al., 2007).

Based on these specifications, a bend sensor is not only advantageous for recording speech-related jaw movements, but also for monitoring oral movements that serve as control conditions in speech production experiments (Sörös et al., 2006a). The use of a chin-mounted bend sensor, however, is limited to speech sounds and oral movements involving the opening of the mouth such as the vowel /a/. Close vowels without considerable mouth opening such as /i/ or mouth movements such as protruding the lips cannot be monitored with a single bend sensor fixed at the chin. Other mounting arrangements of a one degree of freedom sensor or the use of multiple sensors or even a higher degree of freedom device (e.g., ShapeTape, Measurand Inc., Fredericton, NB, Canada) are possible and should be tested.

Bend sensor recordings can also be used as purely behavioral measurements to assess response latencies and accuracies. Bend sensor recordings have already been employed to compare the latency of verbal responses between younger and older adults with the experimental setup presented here (Sörös et al., 2009). Bend sensor recordings are also valuable for assessing responses of patients who, when an overt response is required, perform articulatory movements without vocalization (e.g., in aphasia).

For fMRI data analysis, bend sensor recordings could help to improve the predicted hemodynamic response function that is used for statistical analysis based on the general linear model by incorporating the actual timing of movements and by omitting missing responses. In addition, bend sensor recordings may be included in the linear model as covariate of no interest (confound explanatory variable). Covariates of no interest are expected to account for part of the data variance without being used for statistical contrasts. Covariates of no interest were used, e.g., to model the effect of head motion (Johnstone et al., 2006) and laryngeal motion (Sörös et al., 2008) on fMRI data. In experiments on speech production, bend sensor recordings could be employed to model artifacts associated with jaw movements (Birn et al., 1999). Motion outside the field of view, such as movement of the oral cavity, the sinuses or the pharynx, might cause magnetic field inhomogeneities masking brain activation or generating artifactual intensity changes (Yetkin et al., 1996). For future research, the effect of introducing jaw motion as covariate of no interest in a fMRI analysis should be compared with the effect of other techniques of artifact reduction, such as the use of head motion parameters as covariates of no interest or the removal of artifactual signal components based on independent component analysis (Sörös et al., 2008).

\section{ACKNOWLEDGMENTS}

The authors wish to thank Nicole Baker for developing the data acquisition software used for bend sensor recordings and Dr. Gary Glover for providing his spiral-in/out pulse sequence. The authors also wish to thank two reviewers for insightful comments and suggestions that improved substantially the manuscript. This study was supported by the Heart and Stroke Foundation of Ontario through its Centre for Stroke Recovery, the Ontario Research and Development Challenge Fund and GE Healthcare Canada. 


\section{REFERENCES}

Abrahams, S., Goldstein, L. H., Simmons, A., Brammer, M. J., Williams, S. C., Giampietro, V. P., Andrew, C. M., and Leigh, P. N. (2003). Functional magnetic resonance imaging of verbal fluency and confrontation naming using compressed image acquisition to permit overt responses. Hum. Brain Mapp. 20, 29-40.

Birn, R. M., Bandettini, P. A., Cox, R. W., and Shaker, R. (1999). Event-related fMRI of tasks involving brief motion. Hum. Brain Mapp. 7, 106-114.

Bohland, J.W., and Guenther, F. H. (2006). An fMRI investigation of syllable sequence production. Neuroimage 32, 821-841.

Chambers, J., Bullock, D., Kahana, Y., Kots, A., and Palmer, A. (2007). Developments in active noise control sound systems for magnetic resonance imaging. Appl. Acoust. 68, 281-295.

Christoffels, I. K., Formisano, E., and Schiller, N.O.(2007). Neural correlates of verbal feedback processing: an fMRI study employing overt speech. Hum. Brain Mapp. 28, 868-879.

Di Diodato, L. M., Mraz, R., Baker, S. N., and Graham, S. J. (2007). A haptic force feedback device for virtual realityfMRI experiments. IEEE Trans. Neural Syst. Rehabil. Eng. 15, 570-576.

Edmister, W. B., Talavage, T. M., Ledden, P. J., and Weisskoff, R. M. (1999). Improved auditory cortex imaging using clustered volume acquisitions. Hum. Brain Mapp. 7, 89-97.

Ganesh, G., Franklin, D. W., Gassert, R., Imamizu, H., and Kawato, M. (2007). Accurate real-time feedback of surface EMG during fMRI. J. Neurophysiol. 97, 912-920.

Gorbet, D. J., and Sergio, L. E. (2007). Preliminary sex differences in human cortical BOLD fMRI activity during the preparation of increasingly complex visually guided movements. Eur. J. Neurosci. 25, 1228-1239.
Gorbet, D. J., Staines, W. R., and Sergio, L. E. (2004). Brain mechanisms for preparing increasingly complex sensory to motor transformations. Neuroimage 23, 1100-1111.

Gracco, V. L., Tremblay, P., and Pike, B. (2005). Imaging speech production using fMRI. Neuroimage 26, 294-301.

Graham, S. J., Tam, F., Matenine, D., and Bachmutsky, V. (2009). FMRIcompatible video capture system for kinematic recording. Neuroimage 47, S171.

Jindrich, D. L., Balakrishnan, A. D., and Dennerlein, J. T. (2004). Finger joint impedance during tapping on a computer keyswitch. J. Biomech. 37, 1589-1596.

Johnstone, T., Ores Walsh, K. S., Greischar, L. L., Alexander, A. L., Fox, A. S., Davidson, R. J., and Oakes, T. R. (2006). Motion correction and the use of motion covariates in multiplesubject fMRI analysis. Hum. Brain Mapp. 27, 779-788.

Kleber, B., Birbaumer, N., Veit, R., Trevorrow, T., and Lotze, M. (2007). Overt and imagined singing of an Italian aria. Neuroimage 36, 889-900.

Ku, J., Mraz, R., Baker, N., Zakzanis, K. K., Lee, J. H., Kim, I. Y., Kim, S. I., and Graham, S. J. (2003). A data glove with tactile feedback for FMRI of virtual reality experiments. Cyberpsychol. Behav. 6, 497-508.

Laufs, H., Daunizeau, J., Carmichael, D. W., and Kleinschmidt, A. (2008). Recent advances in recording electrophysiological data simultaneously with magnetic resonance imaging. Neuroimage 40, 515-528.

MacIntosh, B. J., Baker, S. N., Mraz, R., Ives, J. R., Martel, A. L., McIlroy, W. E., and Graham, S. J. (2007). Improving functional magnetic resonance imaging motor studies through simultaneous electromyography recordings. Hum. Brain Mapp. 28, 835-845.
MacIntosh, B. J., Mraz, R., Baker, N., Tam, F., Staines, W. R., and Graham, S. J. (2004). Optimizing the experimental design for ankle dorsiflexion fMRI. Neuroimage 22, 1619-1627.

Murphy, K., Corfield, D. R., Guz, A., Fink, G. R., Wise, R. J., Harrison, J., and Adams, L. (1997). Cerebral areas associated with motor control of speech in humans. J. Appl. Physiol. 83, 1438-1447.

Riecker, A., Ackermann, H., Wildgruber, D., Dogil, G., and Grodd, W. (2000). Opposite hemispheric lateralization effects during speaking and singing at motor cortex, insula and cerebellum. Neuroreport 11, 1997-2000.

Riecker, A., Brendel, B., Ziegler, W., Erb, M., and Ackermann, H. (2008). The influence of syllable onset complexity and syllable frequency on speech motor control. Brain Lang. 107, 102-113.

Seto, E. (2000). Quantifying Head Motion Associated with fMRI Motor Tasks. Ottawa: National Library of Canada.

Seto, E., Sela, G., McIlroy, W. E., Black, S. E., Staines, W. R., Bronskill, M. J., McIntosh, A. R., and Graham, S. J. (2001). Quantifying head motion associated with motor tasks used in fMRI. Neuroimage 14, 284-297.

Smith, A. (1992). The control of orofacial movements in speech. Crit. Rev. Oral Biol. Med. 3, 233-267.

Sörös, P., Bose, A., Sokoloff, L. G., Graham, S. J., and Stuss, D. T. (2009). Age-related changes in the functional neuroanatomy of overt speech production. Neurobiol. Aging. doi: 10.1016/j.neur obiolaging.2009.08.015.

Sörös, P., Cornelissen, K., Laine, M., and Salmelin, R. (2003). Naming actions and objects: cortical dynamics in healthy adults and in an anomic patient with a dissociation in action/object naming. Neuroimage 19, 1787-1801.

Sörös, P., Lalone, E., Smith, R., Stevens, T., Theurer, J., Menon, R. S., and Martin,
R. E. (2008). Functional MRI of oropharyngeal air-pulse stimulation. Neuroscience 153, 1300-1308.

Sörös, P., Sokoloff, L. G., Bose, A., McIntosh, A. R., Graham, S. J., and Stuss, D. T. (2006a). Clustered functional MRI of overt speech production. Neuroimage 32, 376-387.

Sörös, P., Tam, F., and Graham, S. J. (2006b). MR-compatible registration of speech-related movements using a bend sensor. Neuroimage 31, S138.

van Duinen, H., Zijdewind, I., Hoogduin, H., and Maurits, N. (2005). Surface EMG measurements during fMRI at 3T: accurate EMG recordings after artifact correction. Neuroimage 27, 240-246.

Yetkin, F. Z., Haughton, V. M., Cox, R. W., Hyde, J., Birn, R. M., Wong, E. C., and Prost, R. (1996). Effect of motion outside the field of view on functional MR. AJNR Am. J. Neuroradiol. 17, 1005-1009.

Conflict of Interest Statement: The authors declare that the research was conducted in the absence of any commercial or financial relationships that could be construed as a potential conflict of interest.

Received: 13 April 2009; paper pending published: 18 June 2009; accepted: 04 March 2010; published online: 22 March 2010.

Citation: Sörös P, MacIntosh BJ, Tam F and Graham SJ (2010) fMRI-compatible registration of jaw movements using a fiber-optic bend sensor. Front. Hum. Neurosci. 4:24. doi: 10.3389/fnhum.2010.00024

Copyright (C) 2010 Sörös, MacIntosh, Tam and Graham. This is an open-access article subject to an exclusive license agreement between the authors and the Frontiers Research Foundation, which permits unrestricted use, distribution, and reproduction in any medium, provided the original authors and source are credited. 